\title{
Histoire scolaire à l'école primaire : configurations et conscience disciplinaires. Retour sur un corpus de recherche.
}

School history in primary school: disciplinary configurations and awareness.

New approach on specific research material.

\section{Catherine Souplet}

\section{OpenEdition Journals}

Édition électronique

URL : http://journals.openedition.org/educationdidactique/3380

DOI : 10.4000/educationdidactique.3380

ISSN : 2111-4838

\section{Éditeur}

Presses universitaires de Rennes

\section{Édition imprimée}

Date de publication : 21 décembre 2018

Pagination : 89-107

ISBN : 978-2-7535-7711-4

ISSN : $1956-3485$

\section{Référence électronique}

Catherine Souplet, «Histoire scolaire à l'école primaire : configurations et conscience disciplinaires. Retour sur un corpus de recherche. », Éducation et didactique [En ligne], 12-2 | 2018, mis en ligne le 21 décembre 2020, consulté le 06 janvier 2021. URL : http://journals.openedition.org/

educationdidactique/3380 ; DOI : https://doi.org/10.4000/educationdidactique.3380 


\title{
HISTOIRE SCOLAIRE À L'ÉCOLE PRIMAIRE : CONFIGURATIONS ET CONSCIENCE DISCIPLINAIRES
}

\author{
RETOUR SUR UN CORPUS DE RECHERCHE
}

\author{
Catherine Souplet \\ maître de conférences en sciences de l'éducation - didactique de l'histoire \\ université de Lille, Théodile-CIREL EA 4354. catherine.souplet@univ-lille3.fr
}

Trois axes sont développés conjointement dans cet article : poursuivre le travail de spécification du concept de conscience disciplinaire, dans la continuité de travaux menés antérieurement par d'autres didacticiens ; reprendre un corpus déjà constitué et en étudier certains éléments en adoptant des choix méthodologiques explicités; formaliser, par l'analyse, des formes de la conscience disciplinaire pour l'histoire scolaire, qui puissent contribuer à enrichir le champ des recherches en didactique de l'histoire.

Mots-clés : conscience disciplinaire, histoire scolaire, didactique de l'histoire, école élémentaire.

School history in primary school : disciplinary configurations and awareness.

New approach on specific research material.

Three topics are jointly developed in this paper: to continue to study and specify the concept of disciplinary awareness, by considering previous researches conducted by other didacticians; to study some parts of research data through methodological choices; to deliver some results about the forms of disciplinary awareness for school history, which could be useful in the scientific field of didactics of history.

Keywords: disciplinary awareness, school history, didactics of history, primary school. 


\section{Catherine Souplet}

On fait de l'histoire pour savoir le passé, et pour apprendre; apprendre le Moyen Âge, les guerres et la Révolution française, apprendre sur les rois, les/nos ancêtres, et sur les gens. En histoire, on fait des contenus, on écoute et on apprend ; on, et le professeur, parlent du passé.

\section{ÉLÉMENTS DE PRÉSENTATION}

En 2007 dans un article du numéro 2, volume 1, de la revue Éducation et didactique, Yves Reuter proposait une présentation du concept de conscience disciplinaire, dans le prolongement d'une publication antérieure (Reuter, 2003). Au-delà d'en donner des éléments de définition, cet article en discutait également la genèse. Selon Yves Reuter, un des intérêts de ce concept réside dans le fait qu'il « peut contribuer à la construction de l'appareillage conceptuel des didactiques en tant que celui-ci participe fondamentalement de leur identité et de leur autonomie » (Reuter, 2007, p. 65). C'est dans une perspective didactique disciplinaire qu'il est repris ici : en l'occurrence, comme le titre le suggère, la didactique de l'histoire. La réflexion développée dans cette contribution espère tout autant enrichir les travaux autour du concept de conscience disciplinaire que proposer des résultats pouvant s'insérer dans les questionnements et travaux de recherches de la didactique de l'histoire.

Avant de poursuivre, et pour plus de clarté, je cite les éléments de définition proposés dans le Dictionnaire des concepts fondamentaux des didactiques à propos de ce concept :

On pourrait définir la conscience disciplinaire comme la manière dont les acteurs sociaux et, en premier lieu, les sujets didactiques - élèves mais aussi enseignants - reconstruisent telle ou telle discipline. Cela nécessite donc de prendre en compte les formes de ces représentations (des disciplines pouvant être structurées de manière différente par les apprenants et les enseignants) ainsi que la plus ou moins grande clarté dont elles témoignent. (Reuter, 2007/2010, p. 41)

Rappelons par ailleurs que ce concept s'inscrit dans la continuité des élaborations théoriques de l'équipe Théodile-CIREL, visant à étudier les disciplines scolaires et leurs modes d'existence.

Depuis les premières présentations de ce concept (Reuter, 2003, 2007), un ouvrage (Cohen-Azria et al. dir., 2013) a permis de formaliser les constats issus d'une recherche consacrée à l'étude des formes de conscience disciplinaire à l'école primaire. C'est à la suite de cette recherche, en en reprenant le corpus, que s'inscrit le présent article, en étudiant, pour l'histoire scolaire, les formes de conscience disciplinaire d'élèves d'école élémentaire. Le titre de l'article proposé ici reprend en partie certains des titres des chapitres de l'ouvrage Conscience disciplinaire, notamment pour ce qui concerne les chapitres consacrés aux analyses disciplinaires : "Français, configurations et conscience disciplinaires", " Mathématiques, configurations et conscience disciplinaire », idem pour les sciences. De fait, le concept de conscience disciplinaire y est arrimé à celui de configuration disciplinaire ${ }^{l}$. En effet, selon les configurations disciplinaires, c'est à dire selon les actualisations de la discipline dans les pratiques effectives (donc, selon les groupes classes, et selon les pédagogies mobilisées), la conscience disciplinaire peut prendre des formes différentes; mais des variations peuvent également être considérées à un niveau plus individuel, c'est à dire selon les élèves d'une même classe.

La seconde partie du titre souligne un aspect particulier de l'analyse menée, à savoir le fait de travailler avec un corpus constitué antérieurement par une équipe de chercheurs dont je ne faisais pas partie, et cela a des incidences sur lesquelles je reviendrai également. Dans ce corpus, une partie des données concernent l'histoire-géographie ; celles-ci ont été insérées dans l'analyse générale qui a permis la formalisation du concept, tout comme dans l'analyse de la hiérarchisation des disciplines par les élèves, mais elles n'ont pas été spécifiquement traitées par une analyse didactique disciplinaire. Le travail restait donc à faire, et peut permettre de contribuer à identifier ce qui caractérise l'histoire scolaire et ses pratiques à l'école élémentaire, en travaillant avec des discours d'élèves ${ }^{2}$.

Avant d'exposer les choix méthodologiques et les constats issus de l'analyse, je commence par poser quelques éléments de contexte tout autant pour évoquer certaines contraintes qui ont influé sur le travail mené, que pour tenter de mettre en lien les résultats avec d'autres déjà existants. Les contraintes renvoient au fait, déjà évoqué, de travailler sur un corpus constitué antérieurement, ce qui crée une situation de recherche particulière ; j'évoque cela pour en souligner les effets. Sont également présen- 
tés les constats issus d'autres travaux qui résonnent avec la notion de conscience disciplinaire en ce qui concerne l'histoire scolaire, autant en vue de rassembler des recherches qui peuvent s'inscrire dans une forme de complémentarité que pour mettre en regard ces constats avec ceux issus de mon analyse.

\section{ENTRER DANS UN CORPUS DÉJÀ CONSTITUÉ}

Étudier un corpus déjà constitué, déjà analysé, avec des résultats diffusés, provoque une situation particulière pour le chercheur. Il me semble que cela implique, consciemment ou non, de s'inscrire dans une histoire, celle d'une équipe de chercheurs, celle d'une recherche, pour en comprendre la genèse, les choix, les résultats. Penser ainsi ne signifie ni vouloir (à tout prix) rester fidèle à ces différents aspects, ni s'en démarquer volontairement, mais travailler à partir de/avec ce qui existe déjà, en tirer profit, le discuter, le prolonger... sans omettre de se situer de façon explicite.

De fait, cette situation offre comme des avantages. En effet, avoir à disposition des objets finis donne d'emblée matière à réfléchir probablement autrement. J'entends par objets finis un corpus déjà construit, les étapes de recueil, d'organisation des données, de transcription et de saisie dans un logiciel ayant déjà été effectuées ; mais cela inclut également des écrits scientifiques mis à disposition (articles, ouvrage) tout comme des réactions à ces écrits, voire des recherches postérieures qui mobilisent le concept étudié. Tous ces documents donnent à voir tout autant des démarches que des résultats.

Pour autant, on pourrait aussi y voir certains inconvénients, entre autre le fait de ne pas participer à des choix structurants de la recherche, c'est-à-dire la définition de la (des) question(s) de recherche, la construction du cadre théorique et de la méthodologie utilisée, ainsi que le choix du public scolaire étudié. Cela prive aussi des échanges, discussions, séances de travail entre chercheurs, durant lesquelles des décisions sont prises, l'analyse se construit et s'affine, les résultats se stabilisent.

Entreprendre une nouvelle analyse de ces données confronte alors à un corpus important, organisé, et à la nécessité de trouver des angles d'attaque, en faisant avec les choix déjà effectués. Ainsi, dans les questionnaires proposés aux élèves, c'est la discipline histoire-géographie, avec le tiret entre les deux termes, qui est mentionnée. Mon choix aurait été autre. En effet, en adoptant un point de vue de chercheur en didactique, je dissocie histoire et géographie, et je m'en explique ici rapidement. Certes, de façon courante, c'est l'appellation histoire/tiret/ géographie qui prévaut dans le système scolaire, et permet(trait) alors de considérer qu'il s'agit d'UNE discipline scolaire, instituée, avec ses modes d'existence spécifiques. Il ne faut pour autant pas perdre de vue que cette appellation découle directement de la période de la $\mathrm{III}^{\mathrm{e}}$ République qui, en formalisant et institutionnalisant les enseignements de l'école primaire, a scellé ce rapprochement initialement porteur d'une visée patriotique. Or, il convient de ne pas naturaliser cet état de fait et, donc, de ne pas le penser comme immuable. André Chervel (1988) souligne que si, à un moment donné, une discipline scolaire s'installe dans une forme plus ou moins stabilisée, cela s'inscrit dans un processus temporel, et que par conséquent une discipline scolaire n'est pas « une masse amorphe et inerte» (p. 88). Toute discipline scolaire se transforme, évolue, peut s'affaiblir, se renforcer, et la façon de désigner la discipline donne à voir des conceptions sous-jacentes. Histoire et géographie sont encore aujourd'hui considérées conjointement par certains chercheurs, en ce qu'elles constituent de façon commune des disciplines traitant du monde social (Audigier, 1995, 2014 ; Grataloup, 2016). Cela pérennise leur association de façon cohérente, mais repose alors sur un soubassement différent que celui initialement envisagé, en passant d'une visée de formation d'un citoyen qui adhère à des valeurs républicaines collectives, à une visée de construction d'un sujet social critique (Tutiaux-Guillon, 2008). Mais histoire et géographie peuvent aussi être considérées distinctement, car chacune permet un rapport au monde spécifique (Reuter, 2014), entre rapport au passé, et rapport aux territoires et aux espaces. Par ailleurs histoire et géographie renvoient à des contenus différents, et à des champs de référence différents. Penser en termes de distinction ne s'oppose nullement à une complémentarité entre ces disciplines, mais interroge ce sur quoi repose leur rapprochement : des postulats épistémologiques? des pratiques? des formations d'enseignants ? un projet politique? Sans avoir de réponse à toutes ces questions possibles, en tant que didacticienne de l'histoire, c'est bien à la discipline scolaire histoire que je m'intéresse et de façon spécifique. 
Revenons au corpus. Au moment de la mise en place de la recherche, les questions posées aux élèves, et leurs réponses, ont été construites à partir de cette appellation « histoire-géographie $»^{3}$. Cela a induit, pour l'analyse présentée ici, une première difficulté méthodologique. Puisque c'est la discipline scolaire histoire qui constitue l'objet de la réflexion, il a fallu trouver une façon de procéder qui permette de désenchevêtrer histoire et géographie (avec tous les biais que cela engendre potentiellement). Ce point sera plus amplement développé dans la partie exposant les choix méthodologiques.

\section{PRENDRE EN COMPTE D'AUTRES RECHERCHES, D'AUTRES RÉSULTATS, D'AUTRES CONSTATS, OU « DE L'ÉTAT - PARTIEL - DE L'ART »}

Lorsqu'il présente le concept de conscience disciplinaire, Yves Reuter (2007, 2013) mentionne les travaux de François Audigier ${ }^{4}(1993,1995)$ et Nicole Lautier $^{5}$ (1997) en ce qu'ils ont eux aussi exploré la façon dont les sujets didactiques (élèves, enseignants) reconstruisent la discipline. Il m'a semblé utile de retourner vers leurs écrits.

Mais d'autres publications proposent également des regards en lien avec la notion de conscience disciplinaire. Je pense notamment à l'ouvrage dirigé par François Audigier et Nicole Tutiaux-Guillon (2004), Regards sur l'histoire, la géographie et l'éducation civique à l'école élémentaire. On y trouve des résultats conséquents sur « les conceptions et représentations » (p. 33) des enseignants, à partir de l'analyse de leurs discours recueillis par des entretiens et par des réponses à des questionnaires. Une autre recherche, plus récente, peut aussi retenir l'attention. Il s'agit d'un projet dirigé par Françoise Lantheaume, au sein du laboratoire Éducation, cultures, politiques (ECP) avec le soutien de l'Ifé-ENS, qui a pour intitulé «Quand les élèves racontent l'histoire nationale. Questions à l'enseignement». Pour ce projet, des récits d'élèves (du CM2 à la terminale) ont été recueillis à partir des deux consignes suivantes : "Raconte l'histoire de France », et " Si tu avais/ vous aviez à résumer en une phrase, une expression ou un mot l'histoire de France, qu'écrirais-tu/ qu'écririez-vous personnellement ? ${ }^{6}$. Selon les axes choisis, l'analyse de ces récits pourrait probablement apporter des indications sur la façon dont les élèves reconstruisent la discipline. Je mentionne ici ces recherches, mais sans les mobiliser nécessairement dans la suite de ma réflexion; en effet ce que je souhaite explorer est la conscience disciplinaire des élèves de l'école élémentaire. Par conséquent, j'écarte (tout au moins provisoirement) les éléments qui ne contribuent pas spécifiquement à éclairer ce questionnement, mais ces travaux pourraient introduire des éléments pertinents que ce soit dans une poursuite du travail entrepris, ou pour enrichir par une dimension complémentaire le questionnement principal.

Je m'attarde plutôt sur quatre documents : les textes, évoqués plus haut, de François Audigier et Nicole Lautier; un dossier de la DEPP ( ${ }^{\circ} 205$, mars 2016), " Histoire-géographie et éducation civique en fin d'école et de collège »; et le rapport de recherche (2016) « Vécu des disciplines scolaires et décrochage ».

Que retenir des constats avancés par Nicole Lautier et François Audigier à propos de la façon dont les élèves « entrent en histoire » (Audigier, 1995, p. 78)?

François Audigier (1993) s'appuie, entre autres, sur une enquête menée par l'INRP entre 1982 et 1985. Cette enquête a mobilisé différentes méthodologies, observations, questionnaires, entretiens, en s'adressant aux élèves mais aussi aux enseignants et aux familles. Arrêtons-nous sur un aspect de ce travail, qui se rapproche fortement de la méthodologie de la recherche sur la conscience disciplinaire, à savoir des questionnaires adressés à des élèves de $\mathrm{CM} 2^{7}$. Ces deux recherches (Conscience disciplinaire, 2013, et Audigier, 1993 avec les données de la recherche INRP, 1982-1985) visent des objectifs similaires : les représentations que les élèves ont des disciplines, en investiguant plus particulièrement du côté des finalités et des contenus, mais aussi des « critères de réussite » (Audigier, 1986, p. 197). Néanmoins, il faut noter une différence importante : dans l'enquête INRP, les élèves choisissaient leurs réponses parmi des items prédéterminés qui leur étaient proposés ; dans la recherche Conscience disciplinaire, les élèves produisent leur propre discours sans intervention de quelque ordre que ce soit. Cela permet, de façon endogène, d'être au plus proche des constructions des disciplines par les élèves.

Les traits forts des constats élaborés par l'analyse des questionnaires de l'enquête de l'INRP montrent que, pour les élèves, l'histoire sert à " connaître la vie des gens autrefois » et à « savoir la vie des 
personnages célèbres ». Pour être bon élève, il faut apprendre ses leçons et bien écouter. Enfin, le cours d'histoire renvoie à l'idée d'apprendre des dates, d'étudier l'histoire de France, et le début de la vie des hommes. Nous verrons que les résultats de l'analyse du corpus Conscience disciplinaire confortent ces lignes fortes, mais apportent des aspects complémentaires et, parce qu'il s'agit des discours des élèves, mettent l'accent sur des éléments qui n'étaient pas présents dans les questionnaires INRP.

Lorsqu'il donne à voir l'analyse des entretiens menés avec des élèves du secondaire (classes de $4^{\mathrm{e}}$, $\left.3^{\mathrm{e}}, 2^{\mathrm{de}}, 1^{\mathrm{re}}\right)$, François Audigier (1995) relève une « appréciation positive » de la discipline, néanmoins modérée par le fait qu'il faille apprendre, « apprendre des dates, des noms, des mots ( (p. 79). Le temps apparaît comme un des organisateurs forts de la discipline, il permet de mettre en ordre en fonction de la «direction de son écoulement »; cela n'implique pas pour autant l'idée d'une continuité puisque, pour les élèves, le souvenir des objets étudiés reste très fragmentaire, sans logique apparente. Cela amène François Audigier à évoquer, pour les contenus mentionnés par les élèves, « un grand étal où chacun fait son marché » (p. 81). Et que fait l'élève dans le cadre de la discipline histoire? Il est attentif, il écoute, il note, il fait les exercices, il apprend, il reproduit. Notons que l'appréciation globalement positive cohabite avec l'idée de l'ennui face à des habitudes de travail répétitives, et face à une attente de sens qui semble se dissoudre. François Audigier relève également le fait que les références à une identité collective ${ }^{8}$, aux Autres, apparaissent peu, ou de façon très vague; mais il souligne sur ce point " la difficulté d'avoir conscience de ces dimensions et encore plus de les exprimer [ce qui] n'est pas aisé à l'âge de [ces] élèves » (p. 86).

Chez Nicole Lautier (1997), qui a aussi travaillé avec des élèves de classes de $4^{\mathrm{e}}, 3^{\mathrm{e}}, 2^{\mathrm{de}}, 1^{\mathrm{re}}$, l'appréciation positive est également dominante, 94,6\% des élèves interrogés expriment un intérêt, plus ou moins fort, pour la discipline; mais cette étude éclaire d'autres aspects que chez François Audigier. Ainsi, l'histoire est majoritairement perçue selon une vision globalisante, l'histoire "c'est tout », « tout ce qui s'est passé avant nous ", « toutes sortes de choses » (p. 42) ; la trame forte de l'histoire, c'est l'évolution (on peut rapprocher cela de l'idée du temps, comme chronologie organisatrice, notée par François Audigier), avec la perception d'une continuité chemi- nant inexorablement vers le progrès. Par ailleurs, l'histoire est liée à une forme de logique, logique dans l'exposition des événements, logique narrative, ce qui peut se traduire par des propos du type « on n'apprend pas l'histoire, ça se comprend» (p. 48). Quant aux fonctions de l'histoire, Nicole Lautier note que pour les élèves, l'histoire sert à acquérir des connaissances, elle donne des éléments de culture. Par contre, tout comme chez François Audigier, la fonction identitaire se limite à l'évocation d'une identité personnelle, sans perception de l'identité de l'Autre, et la fonction civique ne semble guère faire consensus ; aux yeux des élèves, l'histoire « ne prépare pas nécessairement à remplir son rôle dans la société * (p. 55). Enfin, dans son analyse, Nicole Lautier identifie ce qu'elle nomme un rapport soit externe, soit intime à l'histoire, rapport dans lequel semble plus ou moins se jouer une capacité à se situer dans le monde, à se penser dans l'histoire.

Un des autres documents retenus est le dossier $n^{\circ} 205$ de la DEPP, de mars 2016, intitulé « Histoiregéographie et éducation civique en fin d'école et de collège ». Il s'agit là d'une étude de toute autre nature puisque ce document émane du ministère de l'Éducation nationale, de l'enseignement supérieur et de la recherche, et qu'il rend compte d'une évaluation conduite par un organisme institutionnel, le bureau de l'évaluation des élèves de la direction de l'Évaluation, de la prospective et de la performance, avec l'« objectif de faire le point sur les connaissances et les compétences des élèves dans des disciplines [...] en fin d'école primaire et en fin de collège, au regard des objectifs fixés par les programmes d'enseignement » (p. 5). Au-delà du bilan des acquis des élèves, cette évaluation proposait aux élèves un « questionnaire de contexte » afin de recueillir « leurs opinions sur le contexte dans lequel ils étudient » (p. 27). Si nous ne sommes pas au cœur de la conscience disciplinaire, quelques-unes des réponses apportées par les élèves d'école élémentaire méritent intérêt. Ainsi, l'analyse mentionne que pour les élèves, histoire et géographie fonctionnent communément, dans un même ensemble. Par ailleurs, "plus de huit élèves sur dix aiment participer aux cours d'histoire et de géographie » (p. 27), ils apprécient ces deux disciplines (puisque, dans ce texte, elles sont bien évoquées comme "deux disciplines ») au sein desquelles la pression de l'évaluation ne semble pas pesante. Ils déclarent aimer faire « des recherches de documents », mais l'apprentissage des leçons est 
perçu comme une contrainte par un tiers des élèves. Nous retrouvons dans ces constats certains points communs avec ceux de François Audigier et Nicole Lautier.

Dernier document, le rapport de recherche «Vécu des disciplines scolaires et décrochage » $(2016)^{9}$. Cette recherche a également été menée par des chercheurs de l'équipe Théodile-CIREL, consécutivement à celle sur la conscience disciplinaire. S'il convient de distinguer vécu disciplinaire, qui désigne les émotions et les sentiments qu'associent les élèves aux disciplines scolaires, et conscience disciplinaire, les constats avancés à propos du vécu de l'histoire scolaire sont à prendre en considération en ce qu'ils contribuent également à éclairer la façon dont la discipline est perçue par les sujets didactiques. Lorsque, dans ce rapport, les chercheurs formalisent une identité générale de la discipline, l'histoire apparaît comme fermement associée au passé, à ce qui se passe avant, mais offre une image fragmentée (tout comme a pu le relever François Audigier), composée de domaines divers. Par ailleurs, « c'est une matière de l'apprendre [...] et non du comprendre »; l'évaluation nécessite de réviser, retenir, sans nécessairement comprendre. Voilà qui s'oppose aux constats de Nicole Lautier (construits, rappelons-le, avec les déclarations d'élèves de collège et lycée); mais un point convergent est à noter : le fait que l'on aime ou que l'on n'aime pas, de façon assez clivante, ce qui rejoint les rapports à la discipline suggérés par Nicole Lautier. Autre convergence, avec les constats de François Audigier : l'impression d'ennui, de pratiques répétitives, et d'un sens qui fait défaut. Si l'on regarde la façon dont les constats se spécifient selon les niveaux scolaires, un inversement du vécu de la discipline s'opère entre le primaire et le secondaire. Majoritairement, les élèves d'école primaire expriment un rejet de l'histoire, vécue comme une discipline ennuyeuse et difficile à comprendre, et c'est à partir du collège que le vécu de discipline devient comme plus équilibré entre ceux qui aiment et ceux qui n'aiment pas. Cela bouscule les constats de la DEPP selon lesquels la discipline est appréciée des élèves de l'école primaire. Enfin, dernier point qui retient l'attention : dans les propos des élèves, histoire et géographie sont majoritairement distinguées, ce quel que soit le niveau scolaire.

\section{Faire le point}

Des écarts (mais aussi des convergences) se perçoivent entre ces quatre études, ce qui n'a rien d'inattendu puisque de nombreux éléments diffèrent, notamment le type de public scolaire interrogé, entre élèves du primaire et du secondaire pour François Audigier et du secondaire uniquement pour Nicole Lautier, population scolaire allant du primaire au lycée (en incluant les SEGPA et le lycée professionnel) pour le vécu disciplinaire, élèves du primaire et du collège pris séparément pour l'enquête de la DEPP. Les modalités de recueil varient également, entre questionnaires et/ou entretiens. Enfin, la nature des travaux menés se distingue, entre recherche à visée scientifique ou enquête émanant du ministère de l'Éducation nationale et conduite dans un but d'évaluation des performances.

Si, globalement, l'appréciation positive de la discipline semble effective, elle cohabite néanmoins avec l'idée de l'ennui et d'un sens qui fait défaut. Retenons également le clivage que provoque l'histoire dans la perception des élèves : il semble bien que l'on aime ou que l'on n'aime pas cette discipline. La référence au passé domine de façon forte ${ }^{10}$, en lien avec la notion de temps. Autre point marquant : le couple apprendre/comprendre ; on apprend sans nécessairement comprendre, ou on comprend ce qui exonère quasiment d'apprendre. Ces points peuvent constituer des repères de discussion suite à l'analyse du corpus Conscience disciplinaire. Mais avant d'entrer plus finement dans la réflexion sur la conscience disciplinaire en histoire, un premier constat général vient appuyer ces remarques autour d'apprendre/ comprendre. Une recension de ces deux verbes dans les réponses apportées par les élèves aux huit questions analysées dans cette présentation (voir plus loin dans la méthodologie) montre que le verbe apprendre apparaît dans 20,46 \% des réponses, et le verbe comprendre dans $1,90 \%$...

\section{LA CONSCIENCE DISCIPLINAIRE, CONSIDÉRER DES RÉSULTATS DE RECHERCHE DÉJÀ FORMALISÉS}

Les contributions de l'ouvrage Conscience disciplinaire donnent à voir les résultats de la recherche menée et donnent forme au concept étudié ; ce sont des appuis précieux en ce qu'ils permettent 
de comprendre (partiellement) tout ce à quoi on n'assiste pas lorsque l'on prend les données dans l'après-coup. Pour ce qui concerne les choix méthodologiques adoptés par l'équipe au moment de la mise en ouvre collective de la recherche, je renvoie au chapitre les exposant de façon détaillée ${ }^{11}$. Mais ces choix initiaux deviennent des éléments inhérents au corpus. C'est sur cet ensemble que s'inscrivent ensuite mes choix méthodologiques.

Ainsi, deux questions ne sont pas reprises dans mon étude parce qu'elles ont fait l'objet d'une analyse globale (exposée dans un chapitre au sein de l'ouvrage), pour toutes les disciplines : " Quelles matières travailles-tu à l'école? » et «Classe les matières que tu fais à l'école par ordre d'importance. Tu commences par la plus importante jusqu'à la moins importante ». Les réponses des élèves, et les désignations qu'ils utilisent, donnent à voir la façon dont ils discernent « les espaces de travail et les hiérarchies qu'ils établissent entre ceux-ci » (Lahanier-Reuter, 2013 , p. 35). En ce qui concerne l'histoire, il en ressort que cette désignation apparaît 255 fois dans les réponses des élèves, soit dans 89 \% des réponses. Cela place l'histoire au $3^{\mathrm{e}}$ rang dans la hiérarchie des disciplines mentionnées, après les mathématiques et le français ${ }^{12}$, et devant la géographie qui se trouve au $5^{\mathrm{e}}$ rang avec 243 citations. L'histoire est donc une discipline scolaire qui prend une place nettement identifiée aux yeux des élèves. Il est à noter qu'histoire et géographie sont majoritairement citées de façon distincte, comme des entités indépendantes. La désignation « histoire-géographie » n'apparaît que 4 fois. Ces constats font résonance avec le choix théorique, explicité plus haut, de distinguer histoire et géographie en tant que disciplines scolaires.

Parmi les analyses disciplinaires qui figurent dans l'ouvrage, je m'attarde sur celles concernant les mathématiques et les sciences ${ }^{13}$.Ce qui m'intéresse dans ces analyses, ce sont les méthodologies utilisées qui apparaissent comme construites de façon particulière, selon les choix du didacticien. Ainsi, pour les mathématiques, Dominique Lahanier-Reuter prend pour focale l'étude de l'espace de la discipline, en étudiant d'une part la façon dont cet espace est délimité (et distingué par les élèves) et d'autre part la façon dont il est organisé (ou « comment ils [les élèves] s'y retrouvent », p. 62). Pour mener l'analyse, les questions posées aux élèves sont catégorisées selon cette distinction, puis les réponses sont étudiées en fonction de deux modalités articulées de façon complémentaire, d'une part en considérant la globalité des réponses apportées pour chaque question, et d'autre part en isolant l'ensemble des réponses pour chaque élève. Cette deuxième modalité permet d'identifier des "fragments isolés " pris comme « des indices de formes supplémentaires des consciences disciplinaires $»$ (p. 62). Dominique Lahanier-Reuter mène également un travail d'identification de ce qui constitue des repères du cours de mathématiques (objets disciplinaires, activités, cahiers, etc.) en prenant en compte, dans les énoncés des élèves, la façon dont ces repères sont évoqués. Pour cela, elle s'attarde sur les verbes utilisés en distinguant une identification visuelle (voir), orale (parler de), ou renvoyant à un faire (calculer, tracer), mais aussi en remarquant l'usage du « il y a ».

Pour les sciences, Cora Cohen-Azria propose une analyse plus fragmentée, en ce qu'elle ne procède par regroupement principaux (organisation de la discipline, repères de la discipline) comme ce qui est montré par Dominique Lahanier-Reuter. L'analyse proposée explore plusieurs aspects : la dénomination de la discipline, sa place chronologique dans le cursus des élèves, des repères d'identification, les contenus, la question de la perception des sciences dans l'extrascolaire, les conditions de réussite scolaire en sciences, et des faire disciplinaires. Pour mener son analyse, elle s'appuie tantôt sur des données quantitatives, tantôt sur des extraits d'énoncés particuliers d'élèves, mais en ramenant toujours ces éléments à un groupe-classe identifié.

Dans ces deux analyses se retrouvent des lignes communes, de façon indubitable, mais il apparaît clairement que le chercheur construit son analyse de façon personnelle, et explicite sa méthodologie. Bref, une même recherche, un questionnement initial partagé, un corpus commun construit par des choix collectifs, mais des analyses spécifiques par discipline selon les choix du chercheur, plutôt qu'une méthodologie réplicable. Cela permet donc de se saisir à nouveau d'une partie de ce corpus pour une nouvelle analyse disciplinaire qui vient s'ajouter à celles déjà menées.

\section{CHOIX MÉTHODOLOGIQUES}

Un premier problème s'est posé de façon immédiate, il a déjà été évoqué : la formulation des questions reprend l'expression « histoire-géographie». 


\section{Catherine Souplet}

Les réponses obtenues revêtent en soi un intérêt certain, et pourraient faire l'objet d'une analyse quant à la façon dont ces disciplines sont mentionnées par les élèves (dans quel ordre, la façon dont elles sont distinguées dans l'écrit, leur présence ou absence). Mais il se trouve que l'intention de l'analyse menée est centrée sur la discipline scolaire histoire. Pour cela, j'ai isolé dans le corpus les questions relatives à l'histoire-géographie, puis, suite à des lectures successives des réponses données par les élèves, j'ai conservé uniquement ce qui renvoyait à l'histoire. Certes, il n'y a pas de procédure systématique pour faire ce travail qui ne repose que sur une lecture qui reste subjective. Ont été éliminés tous les éléments renvoyant de façon très explicite à la géographie, mais les réponses ne permettent pas toujours un positionnement net. Les énoncés ambigus obligent alors à une décision du chercheur; ainsi lorsque la réponse apparaît difficile à distinguer, mon choix a été de conserver l'énoncé. Voici quelques exemples (ce qui est barré a été supprimé de la réponse).

Des réponses sans, ou peu d'ambiguité :

- « L'histoire à mieux savoir ce qui s'est passé avant que tu naisses. La géographie à mieux savoir situer les pays et d'autres choses »;

- « La géographie sert à comprendre le fonctionnement des continents »;

- « À savoir l'Antiquité, la Préhistoire, le Moyen Âge, les Temps modernes. La géographie sert à savoir tous les pays »;

- «Pour savoir ce qui se passait avant et ce qui se passe dans d'autres pays »;

- « Ça sert à voir comment habitent les préhistoriens d'avant. Et la géographie sert à éduquer la France »;

- « Ça sert à apprendre la Préhistoire »;

- «À apprendre des choses sur les pays, les continents, l'Union européenne. »

Des réponses parfois ambiguës :

- « Quand on va en $6^{\mathrm{e}}$ on doit déjà savoir des choses en histoire et en géographie donc c'est pour ça qu'il faut écouter. » La référence à la géographie est imbriquée dans la réponse, le tout a donc été conservé ;

- «À savoir comment étaient nos ancêtres et comment le temps a évolué, pourquoi dans les grandes villes il y a plus de commerces. » Difficile là aussi de trancher, le tout a donc été conservé ;

- « L'histoire et la géographie servira plus tard à savoir ce qui s'est passé avant ", à nouveau une imbrication dans la réponse ;

- «À savoir des choses sur le Moyen Âge, le passé, à savoir les fleuves, les animaux. » Les deux derniers termes semblent trop éloignés de l'histoire pour être conservés ;

- « À connaître le monde » ${ }^{14}$.

Une fois ce travail effectué, le nouveau document obtenu pouvait être analysé. Pour entrer dans l'analyse, j'ai associé des questions selon trois axes. Le premier, regroupant trois questions, permet d'explorer la question des finalités conférées à l'histoire, en prenant en compte la dimension extrascolaire.

$\mathrm{H} 10^{15}$ : d'après toi, à quoi servent l'histoire et la géographie ?

H 48 : à ton avis, pourquoi fait-on de l'histoiregéographie à l'école?

H 33 : pour toi, dans ta vie en dehors de l'école, quand est-ce qu'il y a de l'histoire ?

Le second axe examine les contenus, les outils et les faire associés à la discipline, éléments qui permettent d'identifier, de caractériser la discipline de façon endogène à partir des pratiques.

H 16 : qu'est-ce qu'on apprend d'important en histoire-géographie?

H 23 : qu'est-ce que tu fais quand tu es en cours d'histoire-géographie?

H 13: comment sais-tu que tu es en cours d'histoire-géographie?

Le dernier concerne la question de l'apprendre en situation. Qu'est-ce qu'apprendre en histoire pour les élèves, et que faut-il faire pour apprendre?

H 41 : qu'est-ce qui est important, selon toi, pour réussir en histoire-géographie ?

H 45: à quoi fais-tu attention en histoire-géographie?

Un autre problème s'est posé, celui des nonréponses. Selon les questions leur nombre est très variable. Par exemple, pour la Question 10, il y a 36 non-réponses alors que pour la Question 45, on arrive à 81 . Le tableau ci-après montre le total de non-réponses pour chaque question analysée. 
Tableau 1. Total de non-réponses par question

\begin{tabular}{|l|c|}
\hline & Non-réponses \\
\hline Q 10 & 36 \\
\hline Q 33 & 66 \\
\hline Q 48 & 50 \\
\hline Q 13 & 53 \\
\hline Q 16 & 62 \\
\hline Q 23 & 52 \\
\hline Q 41 & 47 \\
\hline Q 45 & 81 \\
\hline
\end{tabular}

Dans ces non-réponses, certaines correspondent à des absences physiques d'élèves, mais certaines sont réellement une absence de réponse, l'élève n'a rien à dire (ou ne sait pas quoi dire) à propos de ce sur quoi on le questionne. Cela constitue un fait en soi, à considérer comme un résultat, d'autant que ces non-réponses sont très variables selon les classes; pour certaines, on trouve peu de non-réponses, quelle que soit la question, alors que pour d'autres, la fréquence de non-réponse est importante, quelle que soit la question. Le tableau 2, ci-dessous, révèle ces particularités.

Tableau 2. Total de non-réponses par question et par classe

\begin{tabular}{|l|c|c|c|c|c|c|c|c|c|c|}
\hline Classes $^{16}$ & $\begin{array}{c}\text { Nombre } \\
\text { d'élèves }\end{array}$ & Q 10 & Q 33 & Q 48 & Q 13 & Q 16 & Q 23 & Q 41 & Q 45 & $\begin{array}{c}\text { Total } \\
\text { par classe }\end{array}$ \\
\hline CF+ & 29 & 3 & 2 & 2 & 3 & 3 & 4 & 3 & 5 & 25 \\
\hline MP1- & 19 & 2 & 4 & 3 & 2 & 2 & 1 & 3 & 2 & 19 \\
\hline MP2- & 19 & 1 & 4 & 5 & 4 & 3 & 4 & 4 & 8 & 33 \\
\hline VC+ & 22 & 5 & 0 & 2 & 2 & 3 & 1 & 3 & 5 & 21 \\
\hline VL & 28 & 3 & 4 & 4 & 5 & 6 & 6 & 3 & 3 & 34 \\
\hline VV- & 28 & 4 & 1 & 2 & 4 & 3 & 1 & 3 & 1 & 19 \\
\hline LD1- & 19 & 0 & 9 & 8 & 5 & 6 & 9 & 8 & 9 & 54 \\
\hline LD2- & 24 & 1 & 5 & 1 & 2 & 2 & 1 & 4 & 3 & 19 \\
\hline LS1 & 31 & 4 & 17 & 4 & 6 & 12 & 3 & 4 & 14 & 64 \\
\hline LS2 & 22 & 2 & 11 & 3 & 6 & 6 & 8 & 0 & 8 & 44 \\
\hline LS3 & 20 & 2 & 5 & 2 & 2 & 2 & 5 & 0 & 1 & 19 \\
\hline FREI- & 4217 & 9 & 4 & 14 & 12 & 14 & 9 & 12 & 22 & 96 \\
\hline $\begin{array}{l}\text { Total par } \\
\text { question }\end{array}$ & & 36 & 66 & 50 & 53 & 62 & 52 & 47 & 81 & \\
\hline
\end{tabular}

La méthodologie même de la passation du questionnaire a peut-être influé sur ces non-réponses ; dans la mesure où il a été diffusé en trois moments distincts dans le temps de la classe, il n'est pas impossible que cela ait provoqué un sentiment de répétition, ou encore que la réponse de l'élève ait été influencée par les questions qui ont précédé. Ces aspects mériteraient d'être étudiés plus précisément, notamment en ramenant ces chiffres à des pourcentages selon le nombre d'élèves ${ }^{18}$, et en menant une analyse par classe (ce qui renvoie aux modes de travail pédagogiques), ou par discipline, voire par élève.

Ces précisions méthodologiques étant posées, venons à l'analyse des réponses des élèves, pour tenter de construire une formalisation de la conscience disciplinaire pour l'histoire scolaire.

\section{LA CONSCIENCE DISCIPLINAIRE : À PROPOS DES FINALITÉS CONFÉRÉES À L'HISTOIRE}

L'histoire scolaire est considérée comme porteuse de valeurs, nourrie d'une interprétation du social (Legris, 2014) et nourrissant indubitablement le social en retour; ses finalités sont associées de manière forte à la construction du futur citoyen, responsable, critique, et éclairé, prêt à agir dans le monde contemporain. Est-ce que, au travers de leurs discours et dans la façon dont ils reconstruisent la discipline scolaire histoire avec les attributs qu'ils lui confèrent, de jeunes élèves d'école élémentaire donnent à voir des aspects qui pourraient être en lien avec ces finalités ou une forme d'articulation entre histoire et monde social ? 


\section{Catherine Souplet}

Tout en contribuant, par l'analyse, à construire/ comprendre une facette de la conscience disciplinaire, certaines questions du corpus renvoient potentiellement à cette articulation histoire/monde social et permettent aux élèves d'exprimer à quoi sert l'histoire selon eux, ou encore comment ils identifient de l'histoire dans la sphère extra-scolaire. Ces questions sont au nombre de trois, je les rappelle ici :

H 10 : d'après toi, à quoi servent l'histoire et la géographie?

H 48 : à ton avis, pourquoi fait-on de l'histoiregéographie à l'école?

H 33 : pour toi, dans ta vie en dehors de l'école, quand est-ce qu'il y a de l'histoire?

Commençons par les Questions 10 et 48. On y retrouve la mention de la discipline histoire-géographie, c'est donc à partir du retraitement des réponses, en isolant ce qui a trait de façon spécifique et suffisamment explicite à l'histoire, que l'analyse est menée. Si ces deux questions se rapprochent par leur formulation, elles révèlent néanmoins des aspects différents.

À quoi sert l'histoire ? Massivement, à connaître le passé. Dans les énoncés des élèves, la formulation la plus fréquente est « à savoir ce qui s'est passé », elle apparaît dans 18,15\% des réponses à la Question 10. D'autres formulations proches, qui renvoient à une connaissance du passé de façon générale, sont repérables, comme " apprendre ce qui se passait », " comprendre le passé », " savoir beaucoup de choses sur avant ", " connaître l'ancien temps », « connaître comment c'était autrefois ». De façon cumulée, ces formulations apparaissent dans 28,05\% des réponses. Au total, la référence à cette connaissance du passé revient dans $46,20 \%$ des réponses. Dans le même ordre d'idée, le mot « avant " arrive dans $27,1 \%$ des énoncés des élèves.

Quant aux verbes utilisés, ce sont majoritairement des formes des verbes "savoir » $(42,2 \%)$ et « apprendre » $(28,7 \%)$ qui apparaissent, alors que le verbe « comprendre " n'est présent qu'à hauteur de $4,3 \%$, et « connaître » à 9,9\%. Bref, l'histoire sert à savoir le passé. Ce passé peut parfois être spécifié par des désignations de contenus, mais sans que ces spécifications ne soient très fréquentes :

- des époques, les plus fréquemment citées étant le Moyen Âge, la Préhistoire, l'Antiquité ;

- parfois des personnages, tels que Charlemagne, Jeanne d'Arc, ou des entités collectives, les gaulois, les hommes préhistoriques ;
- ou encore quelques thématiques, comme "savoir comment mangeaient les hommes », « où ils habitaient $»$.

La mention des « ancêtres » est également présente 24 fois dans les réponses à cette Question 10 . Toutefois, cette référence aux « ancêtres » peut laisser supposer un autre aspect. Il semble que dans certains énoncés, les élèves s'incluent dans la perception qu'ils ont de l'histoire, que ce soit en tant qu'individu isolé ou en tant qu'individu appartenant à un groupe social. Ainsi, certains écrivent que "l'histoire sert à connaître nos ancêtres », le « nos » pouvant renvoyer à une forme d'appartenance. Dans le même ordre d'idée, on trouve des énoncés comme « apprendre ce qui s'est passé avant que nous soyons nés », " savoir les gens sur terre avant nous », " savoir ce qui se passe avant que tu naisses ». Deux grandes tendances se dessinent dans ce type de réponse ; l'élève réfère à lui-même par rapport au repère de sa naissance, ou il fait référence à un passé commun d'une lignée dans laquelle il s'inscrit. De façon diffuse se lit un aspect qui relève d'une forme d'appartenance identitaire. Si l'histoire sert à apprendre le passé, elle peut également permettre de se penser dans un continuum temporel, ou identitaire.

De manière éparse, certains énoncés montrent que l'histoire peut aussi servir "pour plus tard", dans une visée professionnelle (« Pour plus grand ça te servira, pour le métier »), pour le parcours scolaire ( " avoir de bonnes notes», " aller en $6^{\mathrm{e}}$ ), ou encore pour disposer d'une culture générale (« Ça sert à se cultiver »). Un élève écrit aussi : « L'histoire sert à devenir historien ».

Quelques réponses atypiques sont à relever, elles sont mentionnées ici, en ce qu'elles révèlent également une part de ce que peut représenter l'histoire pour de jeunes élèves :

- « L'histoire sert à connaître comment le monde était avant, comment ça se fait que ça a évolué, les gens comment vivaient-ils auparavant »;

- « Grâce à l'histoire on peut imaginer comment les autres gens vivaient $»$;

- "Ça sert à comprendre ce qui s'est passé pendant la guerre, comment ils ont construit les écoles »;

- « L'histoire sert à savoir ce qu'il y avait avant comme les hommes préhistoriques de l'ancien temps et aussi à s'en servir pour dire à d'autres gens qui ne le savent pas »; 
- « L'histoire sert à savoir qui s'est fait tuer par qui. »

Si l'histoire sert à savoir le passé, pourquoi fait-on de l'histoire? Les lectures successives des réponses à la Question 48 ont permis de distinguer différentes dimensions, qui recoupent en partie les constats précédents :

- une dimension identitaire (dans la continuité de ce qui a été mentionné au-dessus), lorsque le discours des élèves laisse penser qu'ils s'incluent dans un groupe social dont il faut connaître le passé, ce qui se traduit le plus souvent par la perspective énoncée de "savoir la vie de nos ancêtres", ou parfois « ce qui s'est passé avant nous »;

- une dimension utilitaire, qui se traduit de deux façons, soit pour le devenir scolaire (« avoir de bonnes notes », "pour la $\left.6^{\mathrm{e}} »\right)$ ou pour un avenir professionnel («pour avoir un bon boulot», «pour être prof »), ou encore " pour plus tard », tout simplement;

- une dimension culturelle, qui renvoie à l'apprentissage de savoirs (en citant des contenus) ;

- une dimension plus générale, selon laquelle l'histoire c'est pour savoir le passé (ce qui s'est passé) sans mention de contenus particuliers, ou tout simplement parce que l'histoire c'est "pour tout " ou « pour apprendre » (où l'on retrouve des constats proposés par Nicole Lautier);

- deux autres dimensions apparaissent plus rarement, une dimension morale pour « ne pas faire les mêmes erreurs » ou «pour devenir égaux en droits », et une dimension mondaine dans le sens où apprendre en histoire c'est pour « avoir de la culture », « pour renseigner une personne qui en a besoin ».

Les dimensions dominantes indiquent nettement que l'histoire, c'est pour avoir des connaissances, des connaissances sur le passé, pour apprendre; cela revient dans quasiment $50 \%$ des réponses. Les dimensions identitaire et utilitaire sont en retrait, dans $11,8 \%$ des réponses pour l'une et $9,2 \%$ pour l'autre.

Donc, on fait de l'histoire pour savoir le passé, et pour apprendre ; et, parfois, cela peut servir à autre chose... Finalement, en dehors de l'école, quand est-ce qu'il y a de l'histoire ? Tel est l'énoncé de la Question 33. Notons que dans les questionnaires proposés aux élèves, c'est la seule question formulée avec le mot « histoire » isolé, une autre question identique étant posée pour la géographie. Néanmoins, au-delà de ce constat, il n'est guère possible de mesurer les effets de cette formulation particulière au milieu des autres.

Un pourcentage important de non-réponses est à relever, $21,78 \%$, sans pouvoir distinguer, comme cela l'a déjà été précisé, les non-réponses qui correspondent à une absence physique de l'élève ou à un choix de ne rien écrire, quels que soient les raisons de ce choix. Des variations importantes selon les classes sont à noter, de $0 \%$ de non-réponse pour la classe VC+ (CM2 de milieu favorisé) ou 3,70\% pour la classe VV- (CM2 de milieu très défavorisé) à $50 \%$ ou plus pour LS1 et LS2 (CM1 et CM2 de milieu mixte). La variable de la caractéristique du milieu socio-culturel ne semble donc pas particulièrement déterminante.

Parmi les réponses effectives, 6,75\% d'entre elles se réduisent à "Je ne sais pas» (avec là aussi des grandes disparités puisque 7 classes sur les 13 ont $0 \%$ de «Je ne sais pas »). Des réponses montrent aussi une absence d'histoire en dehors de l'école : «Je n'en fais pas », «Il y en a pas », « nulle part», " pas du tout », « rien », « non »; elles représentent quantitativement 9,70\% des réponses. Signalons là aussi des variations nettes puisque 3 classes présentent un pourcentage entre 20 et $33 \%$ de réponses renvoyant à une absence d'histoire en dehors de l'école. Une classe de CM2 (VC+) se distingue en ce qu'on n'y trouve aucune non-réponse, aucune réponse du type " Je ne sais pas », et aucune mentionnant qu'il n'y a pas d'histoire en dehors de l'école.

Ces variations importantes tendent à confirmer l'articulation entre forme de conscience disciplinaire et configuration disciplinaire selon les classes.

Mais lorsqu'il y a de l'histoire dans la vie en dehors de l'école, où est-ce ? Les réponses peuvent faire mention de lieux, figurent alors les références (mais sans aucun spécification) aux châteaux, aux musées, aux monuments, et à la maison. Parmi ces lieux, ce sont les musées qui apparaissent le plus fréquemment. Quant à la mention "à la maison », elle est à distinguer des devoirs, le mot « maison » est cité de façon isolée, c'est tout simplement à la maison qu'il y a de l'histoire. Toutefois le « en dehors de l'école » est aussi parfois référé aux devoirs scolaires qui apparaissent dans 6,9\% des réponses. 
Tableau 3. Fréquence globale lieux mentionnés en réponse à la Question 33 : pour toi, dans ta vie en dehors de l'école, quand est-ce qu'il y a de l'histoire?

\begin{tabular}{|l|c|}
\hline Musée(s) & $12,2 \%$ \\
\hline Monument(s) & $4,2 \%$ \\
\hline Maison & $3,4 \%$ \\
\hline Château(x) & $3,0 \%$ \\
\hline
\end{tabular}

Les réponses peuvent aussi mentionner des supports ; alors, c'est la télévision qui revient le plus fréquemment, suivie des livres.

Tableau 4. Fréquence globale des supports mentionnés en réponse à la Question 33 : pour toi, dans ta vie en dehors de l'école, quand est-ce qu'il y a de l'histoire?

\begin{tabular}{|l|c|}
\hline Télévision & $14,4 \%$ \\
\hline Livres & $8,9 \%$ \\
\hline Informations & $3,8 \%$ \\
\hline Internet & $3,0 \%$ \\
\hline
\end{tabular}

Ces résultats conduisent à constater que l'histoire est perçue en dehors de l'école pour $65 \%$ des élèves interrogés, toujours avec des disparités fortes selon les classes. Et la présence de l'histoire est essentiellement associée aux musées, à la télévision, aux livres.

La question était posée en début de cette partie de l'analyse d'un lien éventuel entre reconstruction de l'histoire scolaire par les élèves et perception du social ou de finalités d'ordre civique. Finalement, la tendance forte montre une histoire associée fermement au passé, et connue par des époques, des personnages, des traces qui seraient rendues visibles dans les musées, les livres, plutôt que par référence à une actualité du monde social environnant. Pourtant, même si c'est de façon parcellaire, d'autres aspects apparaissent. Ainsi, le fait que l'histoire pourrait permettre aux élèves de se penser dans une continuité temporelle, ou une lignée (avec une référence aux ancêtres, ou en identifiant des repères temporels par rapport à leur naissance). Et l'histoire n'est pas désolidarisée du monde social ; lorsqu'elle est présente dans le monde, c'est à la maison, dans des lieux publics, ou médiatisée par des livres, des émissions télévisuelles.

\section{LA CONSCIENCE DISCIPLINAIRE : IDENTIFIER L'ESPACE DE LA DISCIPLINE}

Après avoir, dans les énoncés des élèves, identifié ce qui renvoie à des finalités conférées à l'histoire scolaire de façon endogène, essayons de comprendre comment les élèves reconstruisent la discipline scolaire au travers des pratiques. Qu'en disent-ils ? Trois questions permettent cette analyse, en vue de comprendre ce qu'ils font en histoire (ce qui renvoie au faire), comment ils identifient qu'ils travaillent en histoire (ou quels outils permettent ce repérage), et les contenus désignés par les élèves. Ce sont les questions suivantes :

H 16 : qu'est-ce qu'on apprend d'important en histoire-géographie?

H 23 : qu'est-ce que tu fais quand tu es en cours d'histoire-géographie?

H 13 : comment sais-tu que tu es en cours d'histoire-géographie ? ${ }^{19}$

Pour reprendre Dominique Lahanier-Reuter, cela permet d'étudier la façon dont l'espace de la discipline est organisé aux yeux des élèves, ou "Comment ils [les élèves] s'y retrouvent » (2013, p. 62).

Pour toutes les classes, ce que l'on apprend d'important en histoire est référé au temps, que ce soit exprimé par la formule « ce qui s'est passé (avant nous, autrefois, il y longtemps...) », ou par d'autres termes tels que "à l'époque », "les dates » (que l'on apprend), «l'ancien temps », ou encore la frise chronologique qui apparaît pour 3 classes. Si l'analyse de la partie précédente a permis de déclarer de façon synthétique qu' « on fait de l'histoire pour savoir le passé, et pour apprendre », ce que l'on apprend d'important, c'est le Moyen Âge, cité dans 10 classes sur 12. Mais quoi d'autre? Massivement, les guerres (de façon générale, ou la guerre de Cent Ans, et plus rarement les guerres mondiales du $\mathrm{XX}^{\mathrm{e}}$ siècle) et la Révolution française. Et quels personnages étudie-ton ? De façon la plus fréquente, les rois (cités comme tels en tant qu'entités, ou nommés plus spécifiquement) et, à nouveau, « nos ancêtres ».

Il faut noter que les hommes sont très présents dans les écrits des élèves, par des expressions diverses : les gens (énoncés comme tels, ou « les gens célèbres », les « gens qui se battaient »), « les hommes qui ont régné ", " la vie des hommes d'avant ", «les hommes du passé », « les personnes d'avant». De fait, il semble bien que les contenus de l'histoire sont 
associés aux hommes dans le passé. Par contre, si le Moyen Âge revient de façon récurrente dans les réponses des élèves, cela ne se retrouve guère du côté des entités ou des personnages; seuls Jeanne d'Arc et Charlemagne sont parfois cités, et deux fois seulement les seigneurs. Ce sont les hommes préhistoriques qui sont le plus cités.

Il s'avère que les réponses révèlent une diversité effective dans les éléments cités, entre citation d'époques historiques, référence aux hommes (personnages historiques, entités ou simple évocation des hommes de façon généralisante), mention d'événements, de notions (comme la société féodale) ou d'« objets » (par exemple la Déclaration des droits de l'homme). Cette diversité apparaît comme un résultat en soi ; l'histoire n'a rien de monolithique dans l'image qu'en construisent les élèves d'école élémentaire, et même si des traits dominants se dégagent, c'est bien une discipline constituée par une grande diversité dans les éléments qui permettent aux élèves de l'identifier. Cela renvoie directement à l'analyse de François Audigier (1993) quant à ce qui référait au cours d'histoire : pas de réponse majoritairement dominante, mais plutôt une grande dispersion.

L'analyse plus fine de ce que l'on apprend d'important a été menée de façon globale sur les réponses, mais aussi en distinguant les classes. Alors, lorsque l'on considère ces réponses selon chaque classe, il est souvent possible d'émettre l'hypothèse d'un thème dominant (voir tableau ci-dessous), qui correspond peut-être à celui étudié au moment de la passation du questionnaire, ou antérieurement.

D'autres aspects, même s'ils apparaissent moins fréquemment, méritent d'être mentionnés. Ainsi, dans ce que l'on apprend d'important, l'histoire est parfois perçue en terme de lien avec le présent ou de processus : « comment la race humaine a évolué et ce qui s'est passé après », «Avant les personnes n'étaient pas comme nous », "que la vie n'était pas aussi facile que maintenant ", " comment la France a pu devenir comme elle est maintenant », " la différence entre maintenant et le passé ». Autre remarque : la présence $\mathrm{du}$ « comment» dans les réponses, qui semble alors induire dans les réponses la perspective d'une compréhension d'un aspect particulier : « comment ça s'est passé avant notre époque », " comment vivaient nos ancêtres ", " ce que faisaient les hommes, comment ils gouvernaient, comment ils vivaient ", " comment on construisait les maisons, l'âge de pierre, tout plein de choses en histoire ». Ce mot, « comment », appa-
Tableau 5. Fréquence globale des thématiques dominantes par classe en réponse à la Question 16 : qu'apprend-on d'important?

\begin{tabular}{|l|l|}
\hline \multicolumn{1}{|c|}{ Classe } & \multicolumn{1}{c|}{ Thématique } \\
\hline VC+ & Guerre de Cent Ans et découvertes \\
\hline CF+ & XVIII ${ }^{e}$ siècle et Révolution française \\
\hline MP1- & Peu précis, « les ancêtres » \\
\hline MP2- & Peu précis, « avant » \\
\hline VL & Révolution française \\
\hline VV- & Varié \\
\hline LS1 & XIXe siècle \\
\hline LD2- & Société féodale \\
\hline LS2 & Guerre de Cent Ans \\
\hline LD1- & Révolution française \\
\hline LS3 & Moyen Âge \\
\hline FREI & Gaule \\
\hline
\end{tabular}

raît dans 27 répliques soit 11,2 \% des réponses effectives, et est souvent associé au verbe « vivre ». Pour une petite partie des élèves, ce que l'on apprend en histoire renvoie à la façon dont vivent les hommes, ou parfois à « la vie de » et dans ce cas on revient à l'utilisation du verbe « apprendre».

Poursuivons en étudiant ce que disent les élèves à propos de ce qu'ils font en histoire. Les verbes constituent des indicateurs du faire des élèves. Parmi ces verbes, certains apparaissent fréquemment, d'autres moins fréquemment tels que " chercher » (2 fois), « écrire » ( 7 fois), « étudier » ( 7 fois), « lire » (4 fois), « participer» (2 fois), « voir» (2 fois). Curieusement, en histoire, on lit peu, ou tout au moins les élèves n'identifient guère la lecture durant les leçons d'histoire, alors que les classes travaillent souvent avec des documents écrits. Et, toujours selon les élèves, on écrit peu, ce qui converge avec des constats proposés par des recherches en didactique de l'histoire. Quant aux verbes les plus cités, ce sont « apprendre » (72 fois), « travailler » (56 fois), « écouter » (36 fois). Loin derrière arrivent " parler » et « regarder » (12 et 13 fois). Bref, de façon rapide, en histoire, on écoute et on apprend ; on retrouve là des liens avec les conclusions de François Audigier. En s'attardant plus spécifiquement sur chaque classe, là encore apparaissent des particularités selon les verbes les plus fréquents, ce qui pourrait laisser supposer des modalités de travail spécifiques, et renforce l'idée de l'articulation entre formes de conscience disciplinaire et configuration disciplinaire. 
Tableau 6. Fréquence globale des réponses à la Question 23 : qu'est-ce que tu fais quand tu es...?

\begin{tabular}{|l|c|c|c|c|c|}
\hline & $\begin{array}{c}\text { Ap- } \\
\text { prendre }\end{array}$ & Écouter & Parler & $\begin{array}{c}\text { Regar- } \\
\text { der }\end{array}$ & $\begin{array}{c}\text { Travail- } \\
\text { ler }\end{array}$ \\
\hline CF+ & 1 & 1 & 1 & 0 & 11 \\
\hline MP1- & 6 & 1 & 0 & 2 & 4 \\
\hline MP2- & 1 & 0 & 5 & 0 & 3 \\
\hline VC+ & 13 & 5 & 1 & 0 & 4 \\
\hline VL & 13 & 6 & 1 & 0 & 3 \\
\hline VV- & 6 & 10 & 1 & 0 & 3 \\
\hline LD1- & 3 & 1 & 0 & 1 & 8 \\
\hline LD2- & 6 & 2 & 1 & 1 & 6 \\
\hline LS1 & 5 & 2 & 0 & 0 & 5 \\
\hline LS2 & 6 & 1 & 0 & 0 & 2 \\
\hline LS3 & 4 & 7 & 1 & 1 & 3 \\
\hline FREI & 8 & 0 & 1 & 8 & 4 \\
\hline Total & 72 & 36 & 12 & 13 & 56 \\
\hline
\end{tabular}

Par ailleurs, des façons différentes d'exprimer ce qui se fait en classe d'histoire sont identifiables. On trouve des réponses formulées avec un verbe, ou un verbe et un substantif, qui désignent une action scolaire (« J'écoute la maîtresse », « Je prépare mon classeur ", "Je suis attentif ", « Je fais des évaluations $» . .$.$) , mais ce qui revient le plus souvent est une$ formulation du type verbe + contenus. Les verbes utilisés sont alors « faire », « apprendre », « travailler ». En histoire, on fait un contenu :

- « Je fais les rois »;

- «On fait Jeanne d'Arc»;

- « Nous travaillons sur Louis XIV»;

- « On apprend sur Napoléon»;

- « Je travaille la $\mathrm{III}^{\mathrm{e}}$ République »;

- «J'apprends la Préhistoire »;

- «On fait la conquête de la Gaule ».

Dernier point qui participe à l'identification de la discipline histoire, les repères qui permettent à l'élève de savoir qu'il est en cours d'histoire. De façon lapidaire, on pourrait dire : on sait qu'on est en histoire parce qu'on parle du passé et par le professeur. Détaillons cette formule.

« On parle » : en effet à nouveau, l'histoire apparaît comme une discipline où l'on parle. «On parle de...», « le professeur parle » ou « dit ${ }^{18}$. L'étude de la fréquence des verbes les plus souvent utilisés montre cela, de façon globale mais aussi par classe puisque pour quasiment chacune d'entre elles, la fréquence la plus élevée concerne les verbes « dire » ou « parler».

Tableau 7. Fréquence globale des verbes en réponse à la Question 13 : comment sais-tu que tu es en cours?

\begin{tabular}{|l|c|c|}
\hline \multicolumn{1}{|c|}{ Q 13 } & Nb. cit. & Fréq. \\
\hline Parler & 69 & $22,8 \%$ \\
\hline Dire & 40 & $13,2 \%$ \\
\hline Travailler & 18 & $5,9 \%$ \\
\hline Voir & 15 & $5,0 \%$ \\
\hline Changer & 14 & $4,6 \%$ \\
\hline Apprendre & 12 & $4,0 \%$ \\
\hline
\end{tabular}

"Le passé ». Les élèves comprennent qu'ils sont en histoire parce que l'on parle du passé, on a à faire avec le temps, plusieurs termes indiquent cela : avant, époque ("à l'époque », "des époques », époque spécifiée), le passé, des dates. En considérant l'ensemble de ces termes référant au passé, leur fréquence dans les réponses est de 21,9\%. Notons également que la référence à la frise chronologique n'apparaît que deux fois, cet outil ne semble pas particulièrement un marqueur de la discipline pour les élèves.

Enfin, le professeur peut aussi être un élément de repérage, pour deux raisons principales : soit parce qu'il « dit » qu'il s'agit d'une leçon d'histoire ou parce qu'il « parle » du passé, soit parce que les élèves changent d'enseignant lorsqu'il y a histoire et cela devient un repère.

Par contre, les outils de travail en classe, cahier, classeur, livre, etc., ne semblent pas constituer un repérage fort. Ils sont cités, mais pas de manière suffisamment significative (en dehors peut-être du cahier et du tableau). Le document, de façon a priori surprenante $^{19}$, n'est cité que 4 fois dans l'ensemble des réponses à la Question 13.

Si cette partie de l'analyse permet de constater que se dessine une image globale de l'histoire plutôt homogène, il n'en reste pas moins qu'apparaissent des spécificités selon les classes. Une remarque doit être soulignée : la discipline histoire est fréquemment associée, dans ses pratiques, à l'usage de documents en classe, documents que l'on lit, et à la frise chronologique comme marqueur disciplinaire (Audigier, Tutiaux-Guillon, 2004), mais il s'avère que ces outils disciplinaires sont rarement cités par les élèves. Cela ne signifie pas pour autant que les 
élèves ne travaillent pas avec des documents en leçon d'histoire, ou qu'il y a une absence de frise chronologique, simplement cela n'est pas identifié par les élèves en tant que tel. Voilà qui mérite d'être pris en considération.

\section{LA CONSCIENCE DISCIPLINAIRE : APPRENDRE EN HISTOIRE}

Deux facettes des formes de conscience disciplinaire des élèves pour l'histoire scolaire ont été proposées et développées : les finalités conférées à la discipline, et la façon dont l'espace disciplinaire est identifié/construit. Un dernier groupe de questions ${ }^{20}$ renvoie à la question de l'apprendre, du point de vue de l'élève, en situation dans des leçons d'histoire. À quoi faut-il faire attention selon l'élève pendant la leçon d'histoire ? Et si on considère que réussir, c'est bien apprendre, alors y a-t-il des conditions pour cela?

De façon assez logique, puisque l'histoire est une discipline où « on parle », " on dit », cela implique qu'il faut écouter. Reprenons la récurrence des verbes utilisés dans les réponses à la Question 45.

Tableau 8. Fréquence globale des verbes en réponse à la Question 45

\begin{tabular}{|l|c|c|}
\hline \multicolumn{1}{|c|}{ Q 45 } & Nb. cit. & Fréq. \\
\hline Écouter & 24 & $7,9 \%$ \\
\hline Apprendre & 18 & $5,9 \%$ \\
\hline Savoir & 16 & $5,3 \%$ \\
\hline Dire & 15 & $5,0 \%$ \\
\hline Comprendre & 12 & $4,0 \%$ \\
\hline Confondre-mélanger & 9 & $3,0 \%$ \\
\hline Lire & 3 & $1,0 \%$ \\
\hline
\end{tabular}

Faire attention en histoire, c'est " écouter », " écouter ce que le professeur dit», mais aussi « apprendre » et "savoir les leçons » (le mot « leçon » est cité dans 6,9\% des réponses), notamment « les dates » (cité dans 15,2\% des réponses).

Il faut pour certains "comprendre ", " bien comprendre » ou " mieux comprendre». Mais le verbe " comprendre » est aussi mobilisé parce que ce n'est pas si simple, et ce pour une seule classe, un CM2 (VC+). Quelques-uns de leurs énoncés montrent cela :
- « Aux détails de faire les choses avant nous. Je pose des questions quand je ne comprends pas »;

- « Je n'arrive pas à comprendre l'histoire parce que le fils qui a un certain âge règne après une autre personne règne »;

- "J'essaie de comprendre, je m'aide de mes documents, il faut toujours se concentrer, pas faire attention à celui qui te parle »;

- «À tout ce qui est mal expliqué et incompréhensible, j'essaie de comprendre, en histoire, c'est un peu difficile car des fois je ne comprends pas je fais aussi attention à tout ce qui concerne l'exercice. »

S’y retrouver en histoire, ça peut être compliqué, il faut aussi faire attention à ne pas confondre ou mélanger les noms, les dates, les époques, il ne faut pas se tromper dans les noms des rois. Par contre, on ne lit pas beaucoup, à nouveau ce n'est pas un point qui caractérise les leçons d'histoire.

L'orthographe est également mentionnée comme un aspect auquel il faut faire attention ; en effet, il faut bien écrire et faire attention « aux fautes d'orthographe ", "à ne pas mal écrire des mots difficiles », « à ne pas [se] tromper en écrivant ». Dernier point à noter, on trouve peu de mentions de contenus dans les énoncés des élèves en réponse à cette question, contrairement aux autres. Mais quand il y en a, surgissent à nouveau le Moyen Âge (5 fois), la Révolution (4 fois), les rois ( 7 fois), les guerres (5 fois).

Si quelques caractéristiques se dessinent autour de ce faire attention, ce n'est pourtant pas si simple aux yeux des élèves. En effet, cette question est celle qui recueille le plus de non-réponses, 81 sur 303 potentielles. Et faire attention en histoire, c'est souvent faire attention à tout, de façon indéterminée ; ce terme apparaît 26 fois.

Pour réussir en histoire, les conditions sont à peu près identiques à celles requises pour faire attention. Il faut "apprendre ", " savoir », « écouter» les « leçons» et les « dates». Car, finalement, pour reprendre deux réponses d'élèves, « Ce qui est important en histoire et géographie c'est de bien écouter ce qu'on fait en classe et on a fait $50 \%$ du travail qu'on doit faire chez nous » et « Il faut bien écouter le cours car ce n'est pas comme les maths où l'on peut avoir des techniques, là il faut apprendre par cœur ». Là encore, lire les textes, lire les documents reste minoritaire : le mot " texte " n'apparaît que 3 fois, et « document » 2 fois. 
Tableau 9. Fréquence globale des verbes en réponse à la Question 41

\begin{tabular}{|l|c|c|}
\hline \multicolumn{1}{|c|}{ Q 41 } & Nb. cit. & Fréq. \\
\hline Apprendre & 84 & $27,7 \%$ \\
\hline Savoir & 78 & $25,7 \%$ \\
\hline Écouter & 69 & $22,8 \%$ \\
\hline Connaître & 29 & $9,6 \%$ \\
\hline Lire & 12 & $4,0 \%$ \\
\hline Retenir & 10 & $3,3 \%$ \\
\hline Réviser & 7 & $2,3 \%$ \\
\hline Demander & 4 & $1,3 \%$ \\
\hline
\end{tabular}

Des éléments de réponses donnent à voir des aspects autres, qui restent parcellaires car peu fréquents dans les énoncés des élèves, mais qui participent des représentations des élèves et qui contribuent néanmoins à dresser une image de la discipline. Ainsi, on retrouve l'articulation avec le monde en dehors de l'école, réussir en histoire peut être lié à ce que l'on apprend, ce que l'on fait hors de la classe : « Il faut toujours apprendre, se renseigner, écouter en classe, il faut s'intéresser pour savoir beaucoup d'informations », « Il faut s'informer, écouter et essayer de comprendre ce qu'il se passe dans le monde en culture ", " Je pense qu'il faut bien apprendre ses leçons (les retenir) et les revoir régulièrement et être attentive aux cours. Avoir beaucoup de culture, et de bonnes notes, lire », « demander aux personnes âgées pour comprendre puis apprendre ses leçons », « avoir une très bonne culture générale et lire beaucoup, apprendre, retenir ", " regarder les émissions c'est pas sorcier quand ça parle de cette matière ». De façon plus isolée, réussir en histoire peut aussi être associé à des savoir-faire tels que bien écrire, apprendre le vocabulaire, savoir les chiffres romains, savoir compter les années.

Dernière remarque à propos de la frise chronologique. Il a été souligné, dans la partie précédente de l'analyse, qu'elle ne semble guère identifiée comme un marqueur de la discipline. Dans les réponses à ces deux questions, «qu'est-ce qui est important pour réussir en histoire-géographie », et « à quoi fais-tu attention en histoire-géographie », la frise chronologique est mentionnée par trois fois, mais dans une seule classe (VV-). Où l'on revient à nouveau sur l'articulation entre configuration disciplinaire et forme de conscience disciplinaire.

\section{PROPOSITION DE SYNTHÈSE}

Des traits forts se dessinent dans l'analyse menée, traduits par la formule lapidaire proposée en incipit : « On fait de l'histoire pour savoir le passé, et pour apprendre ; apprendre le Moyen Âge, les guerres, et la Révolution française, apprendre sur les rois, les/ nos ancêtres, et sur les gens. En histoire, on fait des contenus, on écoute et on apprend ; on, et le professeur, parle du passé. »

Néanmoins, pour éviter que cette formalisation générale ne soit réductrice, il convient de rappeler qu'à maintes reprises des particularités sont apparues au fil de l'analyse, selon des groupes classes (à la fois selon les modes de travail pédagogique et les milieux socio-culturels) mais aussi selon les sujets didactiques considérés individuellement, en effet dans une même classe, des élèves reconstruisent différemment une image d'une même discipline. Ces variations, ces spécificités, si elles ne sont pas ignorées, n'ont pas été approfondies ici, le choix de l'analyse menée fut plutôt d'identifier des lignes fortes, et donc de reconstruire une image globale de ce que l'on peut désigner par conscience disciplinaire de l'histoire scolaire pour des élèves d'école élémentaire, de CM1 et CM2.

Poursuivre le travail autour de ces variations constitue une perspective à entreprendre, tout autant pour mieux circonscrire (par élargissements) les formes de la conscience disciplinaire de la discipline histoire, que pour en étudier les spécificités dans « le rapport qu'établit l'élève avec la discipline concernée » (Reuter, 2007, p. 62). De plus il semble bien que l'on puisse également penser des variations selon les moments du cursus scolaire. En effet, les constats de l'analyse menée concernent des classes de $\mathrm{CM} 1$ et $\mathrm{CM} 2$, et si certaines convergences avec les recherches antérieures de Nicole Lautier et François Audigier concernant le collège et le lycée (voir en début d'article) ont pu être mises en évidence, des écarts sont également manifestes, notamment sur la question d'une logique organisatrice dans la trame de l'histoire, et sur la question du comprendre.

Pour rester sur des propositions de synthèse, quelques aspects méritent d'être soulignés à nouveau. Les modes d'appréhension que les élèves ont de la discipline, et que l'on peut reconstruire par l'analyse de leurs énoncés, mettent en évidence des aspects qui viennent bousculer une image souvent construite autour de marqueurs disciplinaires que 
seraient les documents et la chronologie. En effet, le terme «document » est rarement mentionné dans leurs propos. Les objets avec lesquels ils travaillent ne semblent pas identifiés comme étant des documents ; et en histoire, on lit peu, alors que l'histoire scolaire est identifiée comme une discipline du texte (Audigier, 2014). De façon similaire, la frise chronologique apparaît rarement dans les discours des élèves. Ces points méritent probablement d'être considérés et éclairés plus finement.

Par contre, on écrit peu et là ce n'est pas une surprise (voir notamment Souplet, 2012), même si des travaux en didactique de l'histoire soulignent les potentialités heuristiques de l'écriture pour apprendre en histoire (Cariou, 2012).

Si les élèves citent beaucoup de contenus, il y a peu de références dans leurs propos à ce qui laisserait voir des "modes de pensée ", tel que cela est envisagé par la didactique de l'histoire, à savoir des modalités de travail organisées autour d'un questionnement, de l'analyse de documents, d'un esprit critique; néanmoins, même si les classes peuvent travailler selon ces modalités, c'est probablement un aspect difficile à restituer pour de jeunes élèves.

Une présence inattendue (mais non incongrue) est apparue de façon nette : celle des ancêtres. Cette présence n'est pas si simple à interpréter, entre renvoi éventuel à la construction d'une identité, insertion de soi dans une temporalité ou une lignée humaine, présence de l'Autre, ou tout simplement imprégnation d'expressions circulant dans l'environnement social (nos ancêtres les Gaulois...), les hypothèses restent fragiles. Soulignons par ailleurs que si, dans leurs déclarations, les élèves font peu de liens entre histoire et présent, l'histoire n'est pas déconnectée du monde social.

Enfin, les élèves aiment-ils l'histoire ou pas, tel que cela était mentionné dans les recherches présentées en début d'article (Audigier, Lautier, rapport sur le vécu disciplinaire, enquête de la DEPP) ? Difficile d'apporter des éléments à cette question, et les questionnaires ne sollicitaient pas explicitement cette dimension. Deux points à noter malgré tout : des formes d'implication particulières qui apparaissent dans les déclarations des élèves, mais aussi un nombre important de non-réponses, ce qui mérite réflexion.

\section{CONCLUSION}

C'est bien l'existence d'un corpus déjà constitué qui a initialement motivé ce travail, avec une double perspective.

La première perspective visait à continuer à faire vivre un corpus de recherche déjà existant. Certes les didactiques ont continûment besoin de nouvelles enquêtes, mais l'existant reste probablement insuffisamment exploité, ne serait-ce que parce qu'un corpus peut être lu et relu à l'aune de plusieurs grilles d'analyse, et que l'épaisseur qu'il recèle ne se révèle qu'en l'étudiant encore et encore. Bien sûr, le corpus mobilisé ici, et l'analyse qui en est menée, mériteraient maintenant d'être mis en lien avec des recherches antérieures (ou encore en lien avec une nouvelle enquête à mener). Néanmoins ce travail de cumulativité, qui permet de renforcer un champ scientifique en étoffant des résultats et/ou en élargissant son empan, se construit pas à pas, ou morceau par morceau. Cette contribution espère être un de ces morceaux.

La seconde perspective, et ce corpus offrait une belle occasion pour cela, est de construire une analyse de façon ascendante, en considérant d'abord les discours des élèves pour ce qu'ils donnent à voir d'un espace disciplinaire (qui apparaît en construction) au sein de l'école élémentaire. Articuler les constats émanant de cette analyse avec d'autres recherches menées dans d'autres espaces, ou avec d'autres finalités, ou encore avec des propos d'enseignants.

Enfin, la recherche qui a permis la construction de ce corpus avait pour objectif principal de comprendre comment les élèves d'école élémentaire reconstruisent, dans leurs discours, une ou des image(s) de disciplines scolaires; et les analyses mettent en évidence toute la complexité de ces reconstructions.

Par ailleurs, si la réflexion proposée ici s'inscrit dans le champ de la didactique de l'histoire, c'est pour approfondir le concept de conscience disciplinaire que le corpus a été construit. Par conséquent, une des autres intentions, déclarée en introduction, veut contribuer à étoffer les résultats, les questionnements, les débats autour de ce concept. Il s'agit alors de permettre que la formalisation d'un concept serve tout autant une didactique disciplinaire que des travaux comparatifs. 


\section{Catherine Souplet}

\section{NOTES}

1. La notion de configuration disciplinaire (Lahanier-Reuter et Reuter, 2007) désigne les variations des modes d'actualisation des disciplines selon les espaces (de prescriptions, de recommandations, de pratiques, de représentations...), les niveaux, les filières, les pays, les époques ; ici, c'est plus particulièrement aux variations selon le cursus, les classes et les pédagogies, que cela renvoie.

2. Le corpus est notamment constitué de questionnaires soumis à 303 élèves de classes de 13 classes de CM1 et CM2 dans huit établissements différents (voir pour une présentation précise le chapitre «Questions de méthodes » dans l'ouvrage cité).

3. Seule une des questions ne reprend pas cette expression « histoire-géographie », c'est la suivante : «Pour toi, dans ta vie en dehors de l'école, quand est-ce qu'il y a de l'histoire?» 4. Le titre de sa thèse, Les représentations que les élèves ont de l'histoire et de la géographie. À la recherche des modèles disciplinaires, entre leur définition par l'institution et leur appropriation par les élèves, renvoie incontournablement à la question qui nous intéresse.

5. Deux chapitres, «L'histoire des élèves » et «L'histoire des professeurs », de l'ouvrage À la rencontre de l'histoire peuvent fournir des réflexions en lien avec la conscience disciplinaire.

6. Cette recherche fait l'objet d'une publication, Le récit du commun. L'histoire nationale racontée par les élèves, aux Presses universitaires de Lyon.

7. Cette enquête pluridisciplinaire avait pour objectif principal d'étudier l'articulation CM2-6e .

8. Je mentionne cet aspect notamment parce qu'une des visées attribuées à l'histoire scolaire est bien cette perspective de participer à la construction d'un sentiment d'appartenance à une communauté humaine, par oscillation entre identité personnelle et collective, et par la rencontre de l'Autre qu'est l'homme d'une autre époque, ou d'une autre contrée.

9. Cette recherche fait l'objet d'un ouvrage : Vivre les disciplines scolaires. Vécu disciplinaire et décrochage à l'école (Reuter (dir.), 2016).

10. Cela semble tout à fait cohérent, et sans surprise. Néanmoins, une des visées de l'histoire scolaire consiste aussi à permettre de mieux comprendre le monde contemporain dans lequel évoluent, et évolueront, les élèves. Il ne s'agit pas de connaître le passé uniquement pour lui-même. Or, les références au présent semblent très rares dans les propos des élèves.

11. Hassan R. et Lahanier-Reuter D. (2013). Questions de méthodes. Dans C. Cohen-Azria, D. Lahanier-Reuter et Y. Reuter (dir.), Conscience disciplinaire. Les représentations des disciplines à l'école primaire (p. 19-33). Rennes : Presses universitaires de Rennes.

12. Notons que dans l'enquête INRP (Audigier, 1983), et plus spécifiquement pour les questionnaires renseignés par les élèves de CM2, l'histoire apparait également au troisième rang pour ce qui concerne la matière préférée... après les mathématiques et l'EPS.

13. Si je n'inclus pas l'analyse disciplinaire pour le français, c'est parce qu'elle est menée par plusieurs chercheurs et exposé de façon différente dans deux chapitres. Cela complexifie alors le travail possible de mise en parallèle des analyses.

14. Mais, cette réponse aurait tout aussi bien pu être conservée pour l'histoire ; c'est la référence à une connaissance du monde que j'interprète comme relevant plutôt de la géographie.

15. Le numéro correspond à la place de la question dans l'ordre du corpus général.

16. Le code qui permet d'identifier chaque classe est celui qui a été adopté lors de la recherche initiale ; voir p. 20-21 dans l'ouvrage de référence (2013).

17. Deux classes de la même école sont regroupées, d'où le nombre important d'élèves.

18. Cet aspect fait l'objet d'une analyse en cours, en reprenant les non-réponses des élèves pour toutes les disciplines. 19. Les textes des questions sont reproduits tels qu'ils ont été soumis aux élèves, mais pour l'analyse, le traitement s'est opéré après avoir isolé les propos directement liés à l'histoire, comme cela a été expliqué supra.

20. Ces constats renvoient à des formes de permanence dans les représentations de la discipline histoire ; en effet, l'analyse lexicale qu'Annie Bruter (1995) a menée à partir de textes d'Ernest Lavisse datant de 1884, 1885 et 1911 montre la présence forte de la parole dans les recommandations qui sont données aux professeurs : il s'agit surtout de dire, d'expliquer et d'interroger.

21. Le document est pourtant communément considéré comme un « objet » incontournable pour travailler en histoire.

22. H 41 : qu'est-ce qui est important, selon toi, pour réussir en histoire-géographie ? et H 45 : à quoi fais-tu attention en histoire-géographie? 


\section{RÉFÉRENCES}

Audigier, F. (1986). Les représentations que les élèves de CM2 et de $6^{\mathrm{e}}$ ont de la géographie. Revue de géographie de Lyon, 2, p. 195-199.

Audigier, F. (1993). Les représentations que les élèves ont de l'histoire et de la géographie. À la recherche des modèles disciplinaires, entre leur définition par l'institution et leur appropriation par les élèves. Thèse, université Paris VI.

Audigier, F. (1995). Histoire et géographie : des savoirs scolaires en question entre les définitions officielles et les constructions des élèves. Spirale, 15, p. 61-89.

Audigier, F. (2014). Histoire, géographie, citoyenneté : les moteurs clandestins du sens. Recherches en Didactiques, 18 , p. 9-23.

Audigier, F. et Tutiaux-Guillon N. (dir.), (2004). Regards sur l'histoire, la géographie et l'éducation civique à l'école élémentaire. Lyon : INRP.

Bruter A. (1995). Lavisse et la pédagogie de l'histoire. Enseignement de la représentation et représentation de l'enseignement. Histoire de l'éducation, 65, p. 27-50.

Cariou, D. (2012). Écrire l'histoire scolaire. Quand les élèves écrivent en classe pour apprendre l'histoire. Rennes : Presses universitaires de Rennes.

Chervel, A. (1988). L'histoire des disciplines scolaires. Réflexions sur un domaine de recherche. Histoire de l'éducation, 38, p. 59-119.

Cohen-Azria, C. (2013). Sciences : configurations et conscience disciplinaires. Dans C. Cohen-Azria, D. Lahanier-Reuter et Y. Reuter, (dir.), Conscience disciplinaire. Les représentations des disciplines à l'école primaire (p. 75-87). Rennes: Presses universitaires de Rennes.

Cohen-Azria, C., Lahanier-Reuter, D. et Reute, Y. (dir.) (2013). Conscience disciplinaire. Les représentations des disciplines à l'école primaire. Rennes : Presses universitaires de Rennes.

DEPP-MEN (2016). Histoire-géographie et éducation civique en fin d'école et de collège. Les dossiers de la DEPP, 205.

Hassan R., Lahanier-Reuter D. (2013). Questions de méthodes. Dans C. Cohen-Azria, D. Lahanier-Reuter et Y. Reuter (dir.), Conscience disciplinaire. Les représentations des disciplines à l'école primaire (p. 19-33). Rennes: Presses universitaires de Rennes.

Lahanier-Reuter, D. et Reuter, Y. (2007). Lanalyse de la discipline. Quelques problèmes pour la recherche en didactique. Dans E. Falardeau, C. Fisher, C. Simard, N. et Sorin, N. (dir.), La didactique du français. Les voies actuelles de la recherche (p. 27-42). Québec: Presses de l'Université Laval.

Lahanier-Reuter, D. (2013a), Mathématiques : configurations et conscience disciplinaires. Dans C. CohenAzria, D. Lahanier-Reuter et Y. Reuter (dir.), Conscience disciplinaire. Les représentations des disciplines à l'école primaire (p. 61-74). Rennes: Presses universitaires de Rennes.

Lahanier-Reuter, D. (2013b). Les matières travaillées et leur hiérarchisation par les élèves. Dans C. CohenAzria, D. Lahanier-Reuter et Y. Reuter (dir.), Conscience disciplinaire. Les représentations des disciplines à l'école primaire (p. 35-46). Rennes: Presses universitaires de Rennes.

Lantheaume, F. et Létourneau, J. (dir.) (2016). Le récit du commun. L'histoire nationale racontée par les élèves. Lyon : Presses universitaires de Lyon.

Lautier, N. (1997). À la rencontre de l'histoire. Villeneuve d'Ascq : Presses universitaires du Septentrion.

Legris, P. (2014). Qui écrit les programmes d'histoire? Grenoble: Presses universitaires de Grenoble.

Reuter, Y. (2003). La représentation de la discipline ou la conscience disciplinaire. Dans La Lettre de la DFLM, 32, p. 18-22.

Reuter, Y. (2007). La conscience disciplinaire. Présentation d'un concept. Dans Éducation et didactique, 1(2), p. 57-71.

Reuter, Y. (dir.) (2007, 2010). Dictionnaire des concepts fondamentaux des didactiques. Bruxelles : De Boeck.

Reuter, Y. (2014). Construire la catégorie de discipline scolaire en didactique(s). Dans Linguarum Arena, 5, p. 79-95.

Reuter, Y. (2016). Vécu des disciplines scolaires et décrochage. Rapport de recherche. Villeneuve d'Ascq : Université Lille 3.

Souplet, C. (2012). Apprendre en histoire à l'école élémentaire: analyse didactique de l'activité cognitivo-langagière en classe. Thèse de doctorat, université Lille 3.

Souplet, C. (2016). Entretien avec Christian Grataloup. Dans Recherches en Didactiques, 22.

Tutiaux-Guillon, N. (2008). Interpréter la stabilité d'une discipline scolaire : l'histoire-géographie dans le secondaire français. Dans F. Audigier et N. Tutiaux-Guillon (dir.), Compétences et contenus. Les curriculums en question (p. 117-146). Bruxelles : De Boeck. 